\title{
Detection of Breast Tumour and Speckle Noise Removal using Bilateral Filter and Bivariate Shrinkage
}

\author{
Simi Wilson \\ Department of Electronics and \\ Communication Engineering \\ Dhanalakshmi Srinivasan \\ College Of Engineering, \\ Coimbatore, India
}

\author{
M. Thangamani \\ Department of Electronics and \\ Communication Engineering \\ Dhanalakshmi Srinivasan \\ College of Engineering, \\ Coimbatore, India
}

\author{
E. Konguvel \\ Department of Electronics and \\ Communication Engineering \\ Dhanalakshmi Srinivasan \\ College of Engineering, \\ Coimbatore, India
}

\begin{abstract}
Automated Breast Ultrasound (ABUS) is an image interpretation to detect the breast tumors. Tumor detection has become a challenging task, due to the presence of poor image contrast, speckle noise and irregular tumor shape. The scope of the work is to remove the speckle noise efficiently while preserving important information from the tumor boundaries. Bilateral filter and the Bivariate Shrinkage Function is applied to the automated whole breast ultrasonic image for the removal of speckle noise. A topographic watershed transform is implemented for ABUS image segmentation process where the précised contour of breast tumors is extracted automatically. This segmented lesion extracts various features like GLCM features, Tamura features, MCHOG features and shape features. Binary logistic regression classifier is applied to the selected feature vectors to analyze the tumor and nontumor images.
\end{abstract}

\section{General Terms}

Image processing, breast tumors detection, Segmentation, Feature extraction, classification

\section{Keywords}

Bilateral filter, bivariate shrinkage, Topographic watershed transform, feature extraction, Binary logistic regression.

\section{INTRODUCTION}

Breast cancer is the second leading cause of morality in women. Initial stage of detection and treatment of breast cancer are the ways of reducing the morality rates. Diagnosis and detection of the breast tumour is done by utilizing two popular modalities namely the mammography and breast ultrasound (US). Mammography is the screening tool to examine and locate the breast tumours and it aims to detect the early stage of the breast tumours. The drawback with mammography is high false positive rate (FPR) and they are not sensitive for women with dense breast tissue. So the clinical examiners use the Ultrasound (US) modal for detecting the dense breast tumours. Comparatively the US modal examination is radiation free and makes the patients feel less pain than the mammography modal. This US modal also faces some problems like poor reproducible and operator independent. To overcome these problems and drawbacks the automated whole breast ultrasound (ABUS), an image interpretation modal is developed and proposed for automatic scanning of the whole breast. This ABUS modal is used to reduce the increased cancer detection rates utilizes less operator dependency and greater reproducibility. The 3-D ABUS images can identify and predicts all the suspicious abnormalities while reviewing hundreds of slices and it consumes less amount of time for review process.
Bilateral filtering is applied to the automated whole breast ultrasonic image for the removal of speckle noise. A topographic watershed transform is implemented in the ABUS images to segment the tumour generated candidates, the located positions and also extracts the potential abnormalities. Thus the segmented ABUS images are used to extract various types of features like GLCM features, Tamura features, MCHOG features and shape features. Then the Binary logistic regression classifier applies the vectors of the selected features to analyse the tumour and non-tumour images. In general the initial stage of image processing is the preprocessing, means scaling the images. The noise reducing techniques are followed to reduce the speckle noise of the image and remove the noise data. After the speckle noise is removed, it is segmented into various constituent parts by the segmentation process to extract the multiple features like shape, size, location,etc.Then the classifiers are used in this feature vectors to identify the normal and abnormal images.

The rest of the paper is organized as follows: Section 2 presents the related works and research in specific areas. The proposed approach has been discussed in Section 3. Section 4 presents the simulation results. Section 5 concludes the paper.

\section{RELATED WORK}

Mammography method is used to detect the early stage of breast cancer. Breast cancers abnormality functions are the calcifications and masses. [1] In mammography method the masses appear to be as the granular clusters, but it cannot analyze the accurate detection of the breast cancer. Digital mammogram is proposed for diagnosing the breast cancer at advance stages. This digital mammogram method is used as a supporting tool for the mammography, which is helpful in ease of analysis process and less time consumption for identifications of the abnormal masses.

Modified image segmentation method is applied for MRI scanning in order to detect the brain tumors. A modified probabilistic neural network (PNN) classifier [3] based on the leaning vector quantization (LVQ) along with the images, data analysis and manipulation techniques is also implemented for automatic scanning of brain tumor classification using MRI scans. The modified PNN classifier is estimated by measuring the performance in terms of the computational time, classification accuracies and training performance. Thus the simulation results illustrates that the modified PNN classifier gives an accurate and rapid classification about the detection of brain tumors.

Mammography is a particular case of CT scanning which utilizes the high resolution film and adapts the method of $\mathrm{X}$ ray in detecting the breast tumors. Strength of this method is the low radiation [4]. Thus the mammogram breast cancer 
images are very effective to determine the cells growth and to detect it. Thus the mammogram technique consumes less time to detect the breast cancer when compared to the image processing threshold, edge detection methods.

Brain tumor is an uncontrolled growth of the tissues in the brain. [7] It is the life threatening cancer the occurs in human beings. The detection and the analysis of exact location of the tumor and the type information are the necessary needs in the field of medical imaging. Magnetic reasoning image (MRI) segmentation plays a major role in locating the tumor. The Fuzzy C-Means algorithm is proposed and applied to find the exact location of the tumor and to decide the type of the tumor.

A dominant research of medical processing is the lung cancer and the lung cancer detection suffers from various kinds of problems like false positive results and accuracy. [9] Thus a novel approach methods is implemented for lung cancer detection, in which the segment of the images are segmented by the Gabor filters in preprocessing stage and the sobel edge detector is applied to detect the preprocessed image. A novel approach performs the detection of lung cancer by estimating the mass value and the average gray scale value of the affection region accurately by consuming less time complexity with higher accuracy.

\section{BREAST TUMOUR DETECTION IN ABUS IMAGES USING BINARY LOGISTIC REGRESSION}

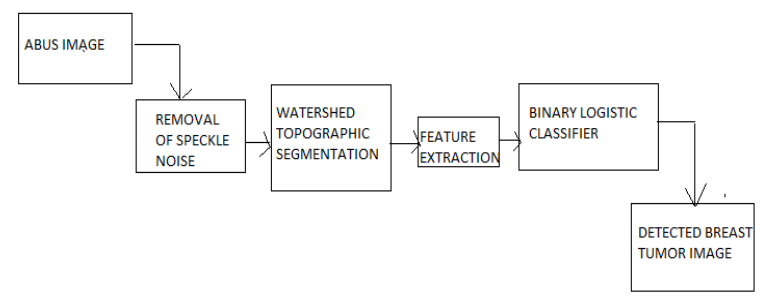

Figure.1: Breast tumor detection and speckle noise removal

\subsection{Removal of speckle noise by Bilateral filtering and Bivariate shrinkage function method}

Normally the biomedical images are affected by various types of noise. These noises should be removed without damaging the desired information is a challenging task. Automated whole Breast Ultrasound (ABUS) images utilize high frequency sound waves and low power consumption to visualize the structure of the breast to observe whether the affected part is harmless, accurate, and noninvasive and the ABUS model is the cost effective method for diagnosis. The quality of the ABUS images like resolution and contrast is degraded due to the presence of speckle noise. The speckle noise diminishes the edge definition, fine details and limits the contrast resolution of the ABUS image. Due to the presence of this speckle noise it is very hard to detect the small and low contrast lesions in the breast. These speckle noise occurs primarily due to the interference of returning wave at the transducer aperture with the dark and bright spots in the images. It also reduces the spatial resolutions and contrast resolutions that are present in the ABUS images. The efficient denoising and filtering technique must be applied to remove the speckle noise and the data corruption from the medical ABUS images. Bilateral filtering and Bivariate shrinkage function is implemented for the speckle noise removal. A bilateral filter is a noise-reducing smoothing filter of the images, edge preserving and non-linear function. Pixel intensity value of the image is replaced with the weighted average. The weight depends upon the radiometric differences such as color intensity, range differences, depth distance, etc. Due to this the sharp edges are preserved any adjusting the weights to the adjacent pixels. Implementation of a simple non-linear bivariate shrinkage function for wavelet de-noising gives the soft thresholding approach, develops an effective and low complexity for adaptive image de-noising. This shrinkage function wants to have some prior knowledge about the noise variance and signal variance for each of the wavelet coefficient. Reduction of noise in the wavelet domain is said to be as the wavelet shrinkage. Coefficient of threshold value selection plays an important role in wavelet shrinkage.

Energy compaction is good in wavelet transforms, it computes the noisy image and applies the threshold values to the detailed sub band coefficients. The effect of the denoising depends upon the selected threshold value of the wavelet thresholding. Smaller threshold values cannot able to eliminate the noise effectively whereas the higher threshold values will gives the noise components and required information. To generate the wavelet threshold coefficients a non-Gaussian brivariate probability function model is implemented. This model captures the dependences between the wavelet coefficients and its parent to improve the denoising method. Thus the Bilateral filter and Bivariate shrinkage function improves the image segmentation and smoothens the homogenous area images while protecting the edges. It also reduces the blocking artifacts by eliminating all the small edges that are amplified by the homomorphic filtering.

\subsection{Tissue segmentation by topography watershed transform model}

After the removal of speckle noise using Bilateral filter in Bivariate shrinkage function the segmentation process is followed. The segmentation is an important process to extract the feature information from the medical image. The input given to the segmentation process is the medical images and the output will be the attributes that are extracted from those images by splitting the images into various constituent parts. A topography watershed transform [10] model is implemented in ABUS images for segmentation that are based on the mathematical morphology operation. This model is applied to the gray level ABUS images for fast analysis, but is cannot manage the process of over segmentation. The topography representation of the image is flooded with water and the watersheds are the divided lines of the domain attraction of rain falling over the region of landscape or topography. The topography watershed transformation is the powerful segmentation process because of its speed, simplicity and complete division of the breast's tissue images. It also provides the facilities in detecting the object boundaries of the images.

\subsection{Extraction of features}

After the composition various types of tissues that were acquired by the topography watershed transform model, the features have to be extracted. They key stage of breast tumor detection and classification is the feature analysis and extraction. Feature extraction methodology extracts the most important features. This segmented lesion extracts various features of the ABUS like GLCM features, Tamura features, MCHOG features and shape features. 


\subsection{Binary logistic regression classifier}

Image classification plays the important role in the digital image analysis. Binary logistic regression classifier is implemented to predict the detection of breast tumour. In this logistic regression analysis method the independent variables are dummy variables and the independent variables consists of the different size levels of the tumours. Thus the binary logistic regression classifier applied the selected feature vectors to analyse the detection of breast tumour and nontumour images. And finally the breast tumour images are detected.

\section{SIMULATION RESULTS Speckle noise removal}

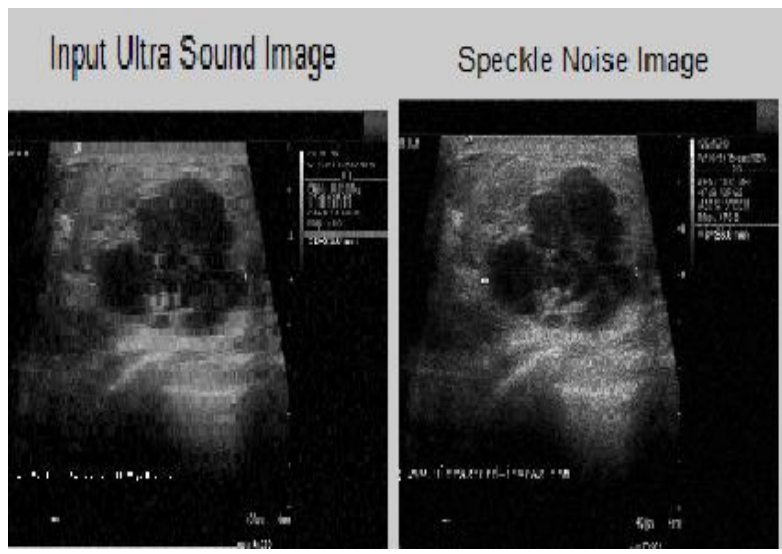

Figure.2 ABUS input image Figure.3 Speckle noise image

In Figure. 2 shows the ABUS input image and it is taken for the preprocessing stage to analyze the presence of Speckle noise as represented in the Figure.3.

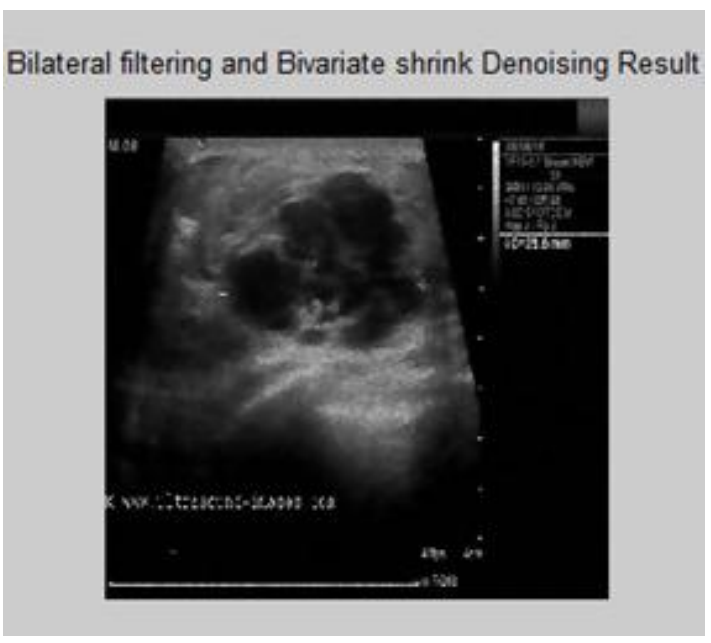

Figure.4 Removal of speckle noise using the

Bilateral filtering and Bivariate shrinkage function

In Figure.4 the bilateral filter removes the speckle noise without reducing the quality of the image. Thus the Bilateral filter and Bivariate shrinkage function improves the image segmentation and smoothens the homogenous area images while protecting the edges. It also reduces the blocking artifacts by eliminating all the small edges that are amplified by the homomorphic filtering.

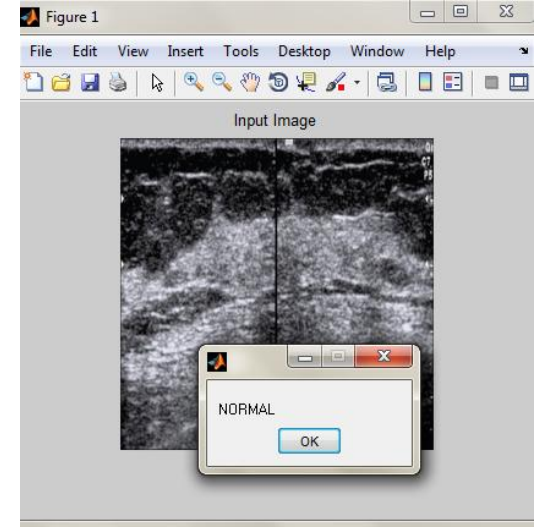

Figure.5 Classification of a normal image

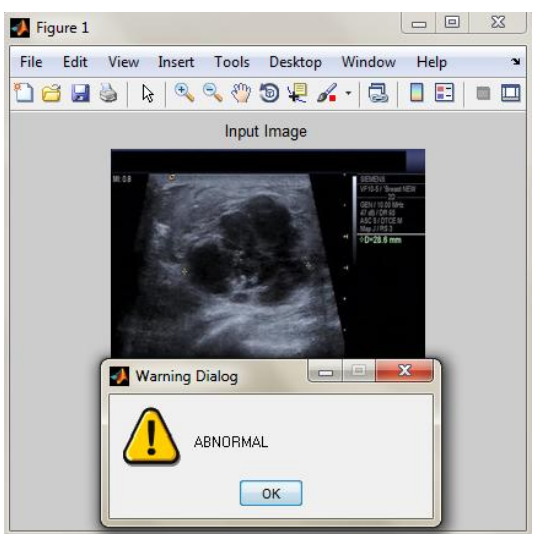

Figure.6 Classification of an abnormal image.

Figure. 5 shows an example image is given as the input. This image is received as the query image and the binary logistic regression classifier classifies it into one of the two predefined categories. The image in figure. 6 is a Ultrasound image of a tumor affected patient and get the output as abnormal.

Table.1 Comparison study of various filter with Bilateral filtering and Bivariant shrinkage function

\begin{tabular}{|c|c|}
\hline FILTERS & PSNR \\
\hline Speckled image & 34.7769 \\
\hline Frost filter & 30.1531 \\
\hline Lee filter & 32.9446 \\
\hline Weiner filter & 22.0738 \\
\hline Anisotropic filter & 38.8026 \\
\hline Bilateral filter & 46.7062 \\
\hline
\end{tabular}

The Speckle noise removal was done by bivariant shrinkage and bilateral filtering. In Table.1 PSNR obtained by various speckle reduction filters tested on two different test images at the same noise variance. Noise Variance $=0.02$. It was found that the Bilateral filtering and Bivariate shrinkage function was very effective in removing speckle noise. 


\section{CONCLUSION}

Bilateral filtering and Bivariate shrinkage function is implemented in the ABUS image to remove the noise and to enhance the tumor boundaries. Then the robust topography watershed segmentation method is utilized for segmenting the breast tumor affected area. More quantitative features are necessary to calculate various databases. Various features like GLCM features, Tamura features, MCHOG features and shape features are extracted for multiresolution analysis and it is useful in processing the datasets from various ABUS systems. The quantitative features of Binary logistic regression classifier predict the output values. The value 0 represents a non tumor image and a value 1 represents a breast tumor image. The weighted neurons are applied in this function to determine the ultimate diagnosis process to predict the breast tumor and its accuracy is biased to analyze the border line cases of the breast tumor. The study can be further extended by using more robust classification method using artificial neural networks.

\section{REFERENCES}

[1] R. F. Chang, K. C. Chang-Chien, H. J. Chen, D. R. Chen, E. Takada,and W. K. Moon, "Whole breast computeraided screening using free-hand ultrasound," in Int. Congr. Ser., Jun., vol. 1281, pp. 1075-1080.

[2] Xiangjun Shi, H.D.Cheng, Liming $\mathrm{Hu}$ (2006), "Mass Detection and Classification in Breast Ultrasound Images Using Fuzzy SVM" in JCIS ,pp 29-36

[3] Dina Aboul Dahab, Samy S. A. Ghoniemy, Gamal M. Selim," Automated Brain Tumor Detection and Identification Using Image Processing and Probabilistic Neural Network Techniques", International Journal of Image Processing and Visual Communication ISSN 2319-1724 : Volume (Online) 1, Issue 2, October 2012.

[4] Nalini Singh, Ambarish G Mohapatra," Breast Cancer Mass Detection in Mammograms using K-means and
Fuzzy C-means Clustering”, International Journal of Computer Applications (0975 - 8887) Volume 22- No.2, May 2011.

[5] Moon.W.K, Lo. C.M, Chang J. M, Huang. C. S, Chen. J. H, and Chang R. F, (2012), "Computer-aided classification of breast masses using speckle features of automated breast ultrasound images," Med. Phys., vol. 39, no. 10, pp. 6465-6473.

[6] A. D. Belsare and M. M. Mushrif, " Histopathological image analysis using image processing techniques: an overview", Signal \& Image Processing : An International Journal (SIPIJ) Vol.3, No.4, August 2012.

[7] Gauri P. Anandgaonkar, Ganesh.S.Sable, "Detection and Identification of Brain Tumor in Brain MR Images Using Fuzzy C-Means Segmentation", International Journal of Advanced Research in Computer and Communication Engineering Vol. 2, Issue 10, October 2013.

[8] Tan .T, Platel. B, Mus.R, Tabar. L, Mann. R.M. and Karssemeijer. N, (2013), "Computer - aided detection of cancer in automated 3-D breast ultrasound," IEEE Trans. Med. Imag., vol. 32, no. 9, pp. 1698-1706.

[9] K.Sankar, Dr.M.Prabakaran, "Region Based Mass Estimation Technique Based Image Segmentation for Lung Cancer Detection Using Gabor Filters", International Journal of Inventions in Computer Science and Engineering ISSN (Online): 2348 - 3539, ISSN (Print): 2348 - 3431 Volume 1 Issue 5 June 2014.

[10] Chung-ming lo, Rong-tai chen, Yeun-chung chang,Yawen yang, ming-jen hung, chiun- sheng huang, and ruey-feng chang, ( 2014) "multidimensional tumor detection in automated whole breast ultrasound using topographic watershed," IEEE transactions on medical imaging,vol. 33, pp. 1503-1511. 